\title{
Neuropsychiatric lupus in Malaysian children: clinical characteristics, imaging features and 12-month outcomes
}

\author{
Sern Chin $\operatorname{Lim}^{1 \oplus}$, Yusma Lyana Md Yusof ${ }^{1 \oplus}$, Bushra Johari ${ }^{2 \oplus}$, \\ Roqiah Fatmawati Abdul Kadir ${ }^{2 \oplus}$, Swee Ping Tang ${ }^{3 \oplus}$ \\ Departments of ${ }^{1}$ Paediatrics, ${ }^{2}$ Radiology, Universiti Teknologi MARA (UiTM) Faculty of Medicine, Sg Buloh, Selangor; ${ }^{3}$ Department of \\ Paediatrics, Pediatric Rheumatology Unit, Selayang Hospital, Selangor, Malaysia.
}

\begin{abstract}
Background. Neuropsychiatric lupus (NPSLE) serves as a marker of severe disease in children with juvenile onset systemic lupus erythematosus (JSLE). This study aims to characterise the clinical and imaging features at diagnosis; and outcomes after 12 months in Malaysian children with NPSLE.

Methods. A retrospective study of all NPSLE patients seen at the Pediatric Rheumatology Unit, Selayang Hospital from January 2004 to May 2017.

Results. Twenty-eight (19.8\%) of 141 JSLE patients had NPSLE with a median presenting age of 10 years (IQR 9 -12), median follow-up of 7 years (IQR 4-11) and female: male ratio of 3.7:1. Twenty-three patients had single episodes of NPSLE and five patients had two distinct episodes each. The mean disease activity score (SLEDAI$2 \mathrm{~K}$ ) was $24.9 \pm 11.8$ at presentation with $81.8 \%$ having high disease activity (score $>12)$. Majority $(60.6 \%)$ present with NPSLE within the first year of SLE diagnosis whilst the remainder occurred at a median of five years (IQR $3-7)$ post-SLE diagnosis. Majority $(75.8 \%)$ had central nervous system (CNS) involvement commonly presenting with seizures, delirium and visual complaints whilst $24.2 \%$ had peripheral nervous system (PNS) involvement. Frequent accompanying features included hypocomplementemia, acute cutaneous lupus and lupus nephritis. Autoantibodies were common; ANA (100\%), anti-dsDNA (78.8\%) anti-RNP (39.4\%) and anti-Sm (39.4\%). Abnormalities were seen in $85.7 \%$ of the magnetic resonance imaging (MRI) studies performed, predominantly supratentorial white matter hyperintensities on T2 images whilst cerebrospinal fluid examination was normal in the majority. All patients with CNS involvement received corticosteroids with immunosuppressive therapy: Cyclophosphamide (20), Rituximab (2). Treatment for PNS involvement included corticosteroids with Azathioprine (6) or Mycophenolate mofetil (2). At 12 months post-NPSLE, majority (85.7\%) recovered without any neurological sequelae.
\end{abstract}

Conclusions. Juvenile-onset NPSLE presents with a myriad of clinical features. It is associated with high disease activity and non-specific MRI features. With early diagnosis and treatment, the majority had good prognosis.

Key words: systemic lupus erythematosus, neuropsychiatric, MRI.

Systemic lupus erythematosus (SLE) is a complex autoimmune disorder characterized by multisystem involvement, including the nervous system. It is defined as juvenile onset SLE (JSLE) when it presents before the age of 18 years which occurs in approximately 10-20\% of all SLE cases. ${ }^{1}$

$\triangle \quad$ Sern Chin Lim

sernchin@gmail.com

Received 3rd December 2021, revised 27th January 2021, accepted 4th April 2021.
Neuropsychiatric SLE (NPSLE) is one of the more complex and poorly understood manifestation of JSLE. $^{2}$ Involvement of the central nervous system (CNS) is more frequent in JSLE and serves as a marker of severe disease in children. ${ }^{2-4}$ The reported incidence of neuropsychiatric symptoms among JSLE patients is between $14-75 \% .^{5}$

The diagnosis of NPSLE is often challenging due to the myriad of symptomatology, many which are non-specific. ${ }^{6}$ Documented NPSLE 
manifestations include headache, seizures, strokes and demyelinating disorders with varying neuroimaging findings. ${ }^{7,8}$ These manifestations are the result of complex pathophysiology affected by genetic, environmental and hormonal factors. It is hypothesized that ethnicity (Asian versus nonAsian) may be one of the potential predictors of neuropsychiatric manifestations and Asian JSLE have a higher risk of developing seizures than non-Asian population. ${ }^{4,9,10}$ However, there is paucity of data of NPSLE in children with SLE in multi-ethnic Malaysia.

This study aims to explore and characterise the various clinical manifestations, laboratory features, imaging findings as well as the outcomes of Malaysian children with NPSLE. We aim to compare our findings with other cohorts of NPSLE children published in existing literature.

\section{Material and Methods}

This is a retrospective observational study of all children diagnosed with juvenile-onset NPSLE (according to ACR/SLICC 2012 NPSLE criteria $^{11}$ ) and managed at the Paediatric Rheumatology Unit, Selayang Hospital, Malaysia from January 2004 till May 2017. This unit is the sole tertiary referral center for all pediatric rheumatology cases in Malaysia until mid-2016, after which an alternative private center was available, thus capturing most JSLE patients in the country during the study period. Subjects were identified from an existing database and data was collected from the hospital's electronic medical records. The diagnosis of NPSLE in the participants were further categorized in accordance with the American College of Rheumatology system for the neuropsychiatric syndromes of SLE. ${ }^{12}$ Patients who did not have at least 12 months of follow-up post diagnosis of NPSLE were excluded from the study.

Data collected included patient demographics; age at onset and duration of SLE illness; clinical features, laboratory findings, treatment and radiological images during the neuropsychiatric episode; assessment of disease activity with Systemic Lupus Erythematosus Disease Activity Index 2000 (SLEDAI-2K index) ${ }^{13}$, and neurological outcomes at 12 months follow-up. Radiological (MRI, MRA, MRV) images were anonymised and reviewed by independent radiologists.

Statistical analysis was done using SAS@ 9.4 software (SAS Institute, Cary NC). The demographic data, clinical, laboratory features and imaging features were analysed using descriptive statistics. Data were expressed as means for continuous variables if normally distributed and median with interquartile range (IQR) if distribution was skewed. Categorical variables are reported as frequencies or percentages. Chi-squared or Fisher exact test was employed for categorical variables and the independent-sample t-test for continuous variables. A $p$ value of $<0.05$ was considered as statistically significant.

This study was investigator initiated and approved by the Malaysian Research and Ethics Committee, National Institute of Health (Approval No: NMRR-19-603-46393) and conducted in accordance with the Declaration of Helsinki. Patient informed consent was waived by our institutional board as only anonymised secondary data was collected.

\section{Results}

Between January 2004 to May 2017, there were a total of 141 patients who fulfilled the criteria for Juvenile Systemic Lupus Erythematosus (JSLE) and had at least 12 months of followup. Of these, 28 patients (19.8\%) had 33 distinct episodes which fulfilled criteria for the diagnosis of NPSLE. Twenty- three patients had a single NPSLE event whereas five patients presented with two separate and distinct episodes of NPSLE throughout their follow-up. The female to male ratio was 3.7 to 1 . Most of the patients with NPSLE were of Malay ethnicity $(79 \%)$ followed by Chinese (17\%), Indian (6\%) 
and mixed ethnicity (3\%). The median age of presentation was 10 years (IQR 9-12) with a range of three to 20 years and the median years of follow-up was seven years (IQR $4-11$ ) with a range of one to 14 years.

Twelve $(36.4 \%)$ of these 33 NPSLE episodes occurred at initial SLE diagnosis with the majority $(60.6 \%)$ of patients presenting within the first year of diagnosis. The remainder of patients presented at a median of five years (IQR 3 - 7) after diagnosis of JSLE. Majority $(75.8 \%)$ had CNS involvement with diffuse neuropsychiatric manifestations whilst $24.2 \%$ had PNS involvement.

The most common neurological manifestation was seizures whilst the predominant physical abnormalities were mental state dysfunction with soft neurological signs (Table I) . There was high anti-nuclear antibody (ANA) and antidouble stranded DNA (anti-dsDNA) antibody positivity (Table I). The most frequent NPSLE diagnoses in our patients was seizures followed by cerebrovascular disease, abnormal behavior and psychoses (Fig. 1). Most patients fulfilled more than one NPSLE categories.

Active lupus manifestations, both clinical and serological, were also commonly seen during the acute presentation of NPSLE (Table I). Majority of patients had high disease activity with a mean SLEDAI-2K score of $24.9 \pm 11.8$ (range 4.0 to 53.0) and those with major neurological symptoms (psychosis, delirium, seizures, abnormal behaviour) exhibited even higher disease activity with a mean SLEDAI-2K score of 31.7 (Table I).

Cerebrospinal fluid (CSF) findings were normal in two thirds of the patients despite the presence of dramatic clinical features. Of the remaining five, three had features of aseptic meningitis with high protein and low glucose whereas the remaining two had isolated elevations of CSF protein. Cerebrospinal fluid IgG screening was performed in three patients, of which only one was elevated and this was a patient with seizures and psychosis. Electroencephalogram (EEG) were performed in seven out of 16 patients who presented with seizures. Four were abnormal with diffuse slow and attenuated background waves reflective of their altered mental states.

The most common abnormality on brain MRI was non-specific white matter hyperintensities $(\mathrm{WMH})$ in the supratentorial regions on $\mathrm{T} 2$ and FLAIR sequences (Table II). None of the presenting features were significantly associated

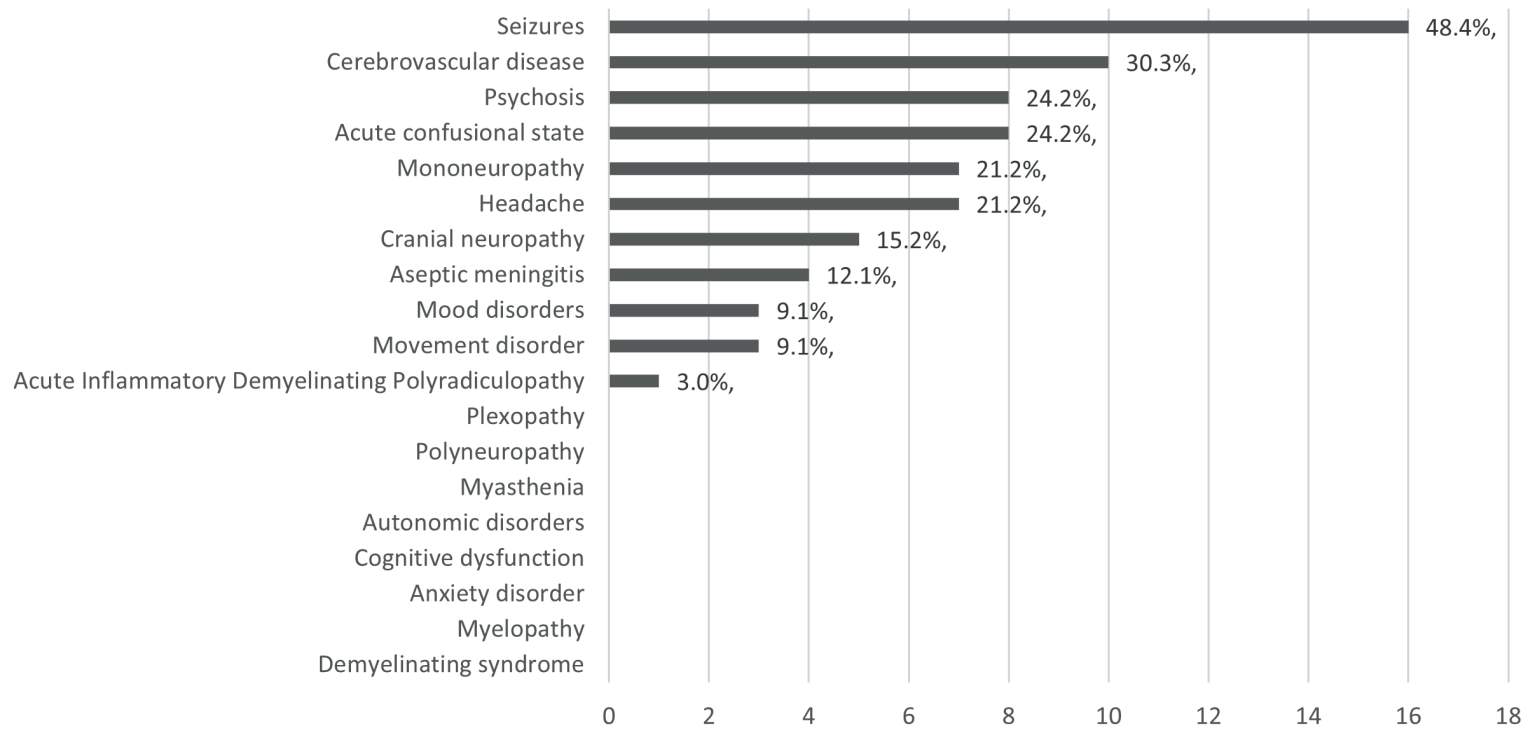

Fig. 1. Frequency of each neuropsychiatric lupus diagnostic category based on the American College of Rheumatology Nomenclature. ${ }^{12}$ 
Table I. Presenting features of patients with neuropsychiatric lupus.

\begin{tabular}{|c|c|c|c|}
\hline Presenting features & $\begin{array}{l}\text { Frequency }(\%) \\
n=33\end{array}$ & Presenting features & $\begin{array}{l}\text { Frequency }(\%) \\
n=33\end{array}$ \\
\hline Clinical features & & $\begin{array}{l}\text { Neurological abnormalities on } \\
\text { examination }\end{array}$ & \\
\hline Seizures & $15(45.5)$ & Abnormal reflexes & 13(39.4) \\
\hline Delirium & $10(30.3)$ & Mental state dysfunction & 13(39.4) \\
\hline Sensory deficit & $9(27.3)$ & Reduced power & 12(36.4) \\
\hline Visual problems & $9(27.3)$ & Abnormal tone & $6(18.2)$ \\
\hline Headache & $8(24.2)$ & Fundoscopic abnormalities & $6(18.2)$ \\
\hline Psychosis & $8(24.2)$ & Cranial nerve abnormalities & $6(18.2)$ \\
\hline Abnormal behavior & $8(24.2)$ & Cerebellar signs & $4(12.1)$ \\
\hline Weakness of limbs & $6(18.2)$ & Sensory deficit & $4(12.1)$ \\
\hline Mood disorder & $4(12.1)$ & & \\
\hline Movement disorder & $3(9.1)$ & & \\
\hline Others & $3(9.1)$ & Disease activity (SLEDAI-2K scores) & \\
\hline Photophobia & $1(3.0)$ & High disease activity $(>12)$ & $27(81.8)$ \\
\hline Hearing problems & $1(3.0)$ & Moderate disease activity (6-12) & $5(15.2)$ \\
\hline Developmental regression & $0(0)$ & Low disease activity $(<6)$ & $1(3.0)$ \\
\hline Lupus manifestations in other organs & & Immunologic markers & \\
\hline Low complement & $28(84.8)$ & ANA & $33(100.0)$ \\
\hline Thrombocytopenia & $15(45.5)$ & Anti dsDNA & $26(78.80)$ \\
\hline Acute cutaneous rash & $15(45.5)$ & Anti Sm & $13(39.4)$ \\
\hline Renal & $14(42.4)$ & Anti RNP & $13(39.4)$ \\
\hline Leukopenia & 13(39.4) & Anti Ro & $10(30.3)$ \\
\hline Positive direct coombs & 10(30.3) & Antiphospholipid & $8(24.2)$ \\
\hline Oral ulcer & $9(27.3)$ & Anti La & $7(21.2)$ \\
\hline Alopecia & $7(21.2)$ & Anti Jo1 & $2(6.1)$ \\
\hline Chronic cutaneous rash & $6(18.2)$ & & \\
\hline Serositis & $5(15.2)$ & & \\
\hline AIHA & $2(6.1)$ & & \\
\hline Synovitis & $2(6.1)$ & & \\
\hline
\end{tabular}

AIHA: Autoimmune hemolytic anemia

SLEDAI-2K: Systemic Lupus Erythematosus Disease Activity Index 2000

ANA: Anti-nuclear antibody

Anti dsDNA: Anti-double stranded DNA antibody

Anti Sm: Anti-Smith antibody

Anti RNP: Antinuclear ribonucleoprotein antibody

Anti Ro: Anti-Ro antibody

Anti La: Anti-La antibaody

Anti Jo1: Anti-Jo1 antibody

with MRI changes ( $\mathrm{p}>0.05)$ but abnormal MRIs were commonly found in those with delirium, psychosis, seizures or abnormal behavior (Fig. 2). There was also no significant correlation between patients with cutaneous vasculitis or any of the NPSLE syndromes with the brain
MRI changes $(\mathrm{P}>0.05)$. The abnormalities on magnetic resonance angiography (MRA)/ magnetic resonance venography (MRV) were venous infarction (1), sagittal sinus venous thrombosis (1) and irregularities of both middle cerebral arteries (2) (Table II). 


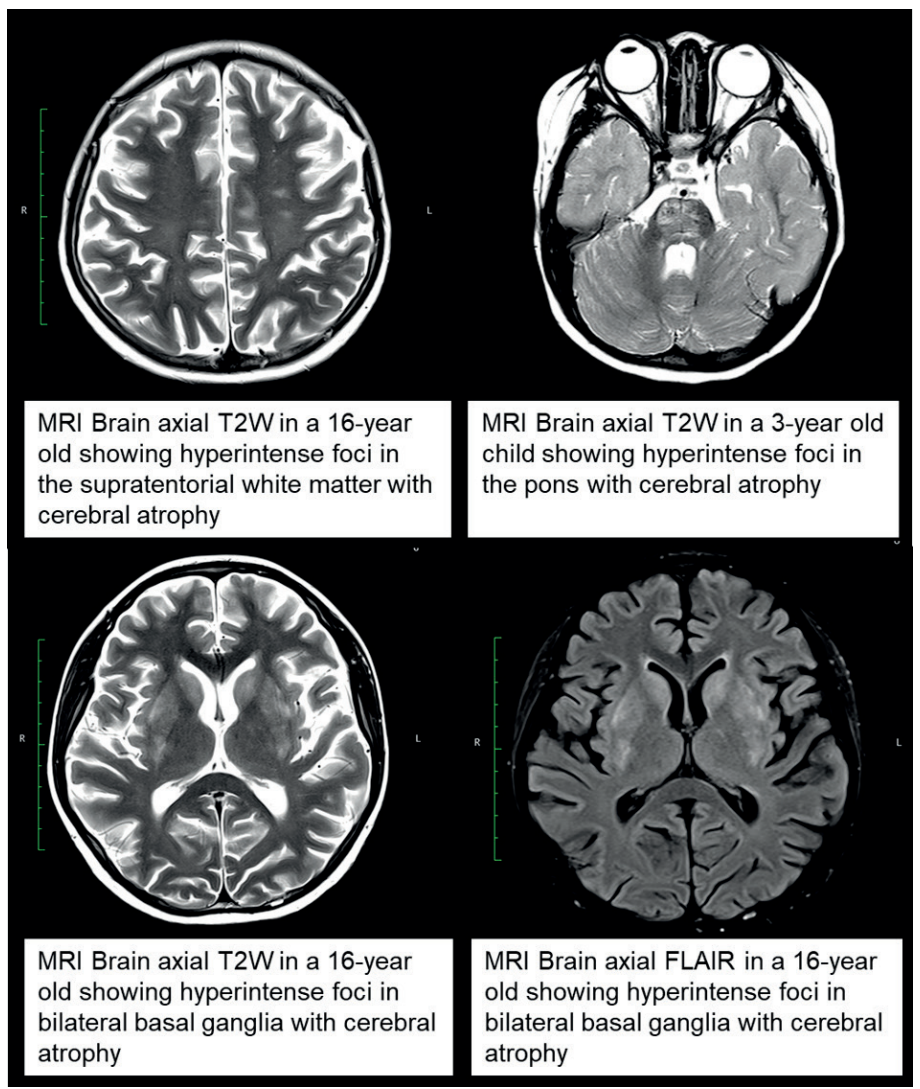

Fig. 2. Magnetic resonance images of the patients with neuropsychiatric lupus.

Table II. Magnetic resonance imaging and magnetic resonance angiography/venography findings in patients with neuropsychiatric lupus.

\section{Frequency}

MRI ( $\mathrm{n}=28)$

Normal 4

Abnormal 24

\begin{tabular}{lccccc}
\hline Sites with abnormalities & $\begin{array}{c}\text { Supratentorial } \\
\text { white matter } \\
(\mathrm{n}=17)\end{array}$ & $\begin{array}{c}\text { Brainstem } \\
(\mathrm{n}=5)\end{array}$ & $\begin{array}{c}\text { Cortical grey } \\
\text { matter } \\
(\mathrm{n}=3)\end{array}$ & $\begin{array}{c}\text { Basal ganglia } \\
(\mathrm{n}=4)\end{array}$ & $\begin{array}{c}\text { Cerebellum } \\
(\mathrm{n}=2)\end{array}$ \\
$\begin{array}{l}\text { Types of abnormalities } \\
\text { Signal change abnormalities }\end{array}$ & 17 & 5 & 2 & 3 & 2 \\
$\quad$ T2 hyperintensities & 16 & 5 & 3 & 4 & 2 \\
$\quad$ FLAIR hyperintensities & 1 & & & & \\
\hline Parenchymal defect & 11 & & & & \\
Atrophy & & & & & \\
\end{tabular}

MRA/MRV ( $\mathrm{n}=5)$

Normal

Abnormal

MRA abnormalities 2

MRV abnormalities 1

MRI: magnetic resonance imaging, MRA: magnetic resonance angiography, MRV: magnetic resonance venography 
All patients received pulsed intravenous Methylprednisolone during their acute presentation. Of the 28 patients, all 20 who had CNS involvement were treated with intravenous Cyclophosphamide and 2 of these patients received two separate courses of 12 monthly intravenous Cyclophosphamide for each of their separate NPSLE episodes. Additionally, two patients on Cyclophosphamide received Rituximab as well. Of the eight patients with PNS involvement, two patients were treated with Mycophenolate mofetil while the other six received Azathioprine and one patient received additional intravenous Immunoglobulin (IVIG). All 16 patients with seizures required antiepileptic drugs and three patients received antipsychotic drugs. One patient had anticoagulant therapy for antiphospholipid syndrome with multiple cerebral sino-venous thrombosis.

At 12 months post diagnosis of NPSLE, 24 out of 28 patients $(85.7 \%)$ had complete recovery with no neurological deficits despite initial high disease activity. Two patients (7.1\%) had mood disorders, one patient (3.6\%) developed epilepsy and one patient (3.6\%) had both residual dystonic tremors and cortical blindness. In patients with two episodes of NPSLE, $60 \%(3 / 5)$ had complete recovery. There were two deaths $(7.1 \%)$ in this cohort; both from infections but were unrelated to the treatment given for NPSLE.

\section{Discussion}

Our paper is the first to highlight characteristics of 28 children with NPSLE in Malaysia amongst a heterogenous South Asian population, contributing to global knowledge of pediatric NPSLE and helping to advance our understanding of the genetic influence on the lupus manifestations and response to therapy. Our findings are consistent with other studies which show a prevalence of NPSLE between 14$25 \%$ with the majority occurring in the first year of the disease. ${ }^{7,9,14,15}$
Neuropsychiatric lupus is a complex entity with broad range of categories under the American College of Rheumatology criteria. ${ }^{12}$ This clinical diversity makes accurate assessments difficult in both adult and pediatric studies. ${ }^{4,6,7,76}$ The most common reported clinical manifestations are diffuse neuropsychiatric syndromes and CNS involvement which is similarly seen in our cohort. ${ }^{3,4,6,14,17}$

Seizures featured predominantly in our cohort and this high prevalence of seizures was similarly seen in other studies involving Asian children. ${ }^{3,4,9,10}$ On the contrary, non-Asian children tend to present more with headaches, cognitive dysfunction and psychosis. ${ }^{6,716,18}$ These major neurological manifestations were also associated with higher overall disease activity which supports the current understanding that NPSLE is an immunologically and inflammatory active state although no single immunological pathway has yet to be identified. ${ }^{2,19,20}$ The presence of cutaneous vasculitis did not correlate with major NPSLE syndromes (apart from cranial neuropathy) in our study and this lends further support to the growing body of evidence that vasculitis may not have a prominent role in the pathogenesis of NPSLE. ${ }^{20,21}$

Anti-phospholipid antibodies have been reported to be associated with distinct NSPLE syndromes such as movement disorders, seizures and cerebrovascular disease but these associations were not identified in our patients. ${ }^{7}$ However, Anti-dsDNA, Anti-Smith (Sm) and Antinuclear ribonucleoprotein (antiRNP) antibodies which have been reported to be strongly associated with NPSLE syndromes, were similarly found in high frequencies in our patients. ${ }^{19}$ Both Anti-dsDNA and Anti-RNP antibodies have been shown to have a possible correlation with seizure disorders. Studies have shown that these antibodies can cross the disrupted blood brain barrier in patients with SLE and demonstrate cross-reactivity with anti$\mathrm{N}$-methyl-D-aspartate receptor and neuronal surface antigens; causing neurotoxicity and inducing neuronal death. ${ }^{2,22}$ 
Interestingly, despite severe and often dramatic clinical symptoms, CSF examination was normal in two thirds of the 14 samples. Even when abnormal, the changes were mild with no specific correlation to clinical manifestations. Thus, CSF examination does not seem to provide any additional information to support the diagnosis of NPSLE; but is nevertheless important to exclude CNS infections. ${ }^{20,22}$

Magnetic resonance imaging abnormalities were seen in more than $80 \%$ of our cohort in contrast to prevailing studies which showed predominantly normal MRIs for both adult and pediatric NPSLE series (42-59\%)., ${ }^{7,16,20,22-24}$ The most common abnormality in our study were white matter hyperintensities which is also reported in other studies. ${ }^{20,22-25}$ These changes may suggest small vessel disease or vasculitis, but their specificity has yet to be established as these lesions have also been reported in patients with SLE and no neuropsychiatric findings. ${ }^{23} \mathrm{We}$ could not determine any correlation between NPSLE syndromes with specific MRI findings in our study due to the small number of subjects but no specific correlation has been found either in other published studies. ${ }^{20,22,23}$ In our experience, even patients with severe clinical symptoms such as psychosis or seizures may not demonstrate any abnormalities on MRI.

The most common EEG change in our patients were diffuse slow and attenuated background waves, consistent with other studies and suggestive of a diffuse immunopathological process in NPSLE. ${ }^{26}$ None showed any epileptiform discharges despite the occurrence of seizures but most of our EEG recordings were delayed due to resource constraints.

Corticosteroids is the mainstay of therapy for SLE and plays a crucial role in the acute management of NPSLE. While there is currently no true consensus on the optimal therapeutic regime for Juvenile onset NPSLE; clinical experience seems to point to the efficacy of corticosteroids in combination with Cyclophosphamide. $18,20,24,27$ Other modalities such as Rituximab, IVIG and even plasmapheresis have been reportedly used when there is poor response. ${ }^{20}$ However, the evidence for these treatments remain largely anecdotal with reports mainly from small clinical cohorts or case reports. ${ }^{20,24}$ Mycophenolate mofetil and Azathioprine are frequently used in mild NPLSE and also recommended for maintenance therapy for all patients ${ }^{20,24,27}$ Anticoagulation and antiplatelet therapy is recommended for any thrombotic process. ${ }^{24,27}$ Patients may need other supportive therapies such as anti-epileptic drugs or psychiatric medications during the acute episode..$^{20,24,27}$ While there remains a scarcity of randomized controlled studies due to the rarity of this disease particularly in children coupled with the heterogeneity of disease presentations, there are international guidelines to assist with the management of this difficult condition. ${ }^{27}$

Our study is too small to offer any specific therapeutic approach but our cohort appears to have had good response to the combination of steroids and intravenous Cyclophosphamide or Rituximab. The overall survival rate and absence of permanent neuropsychiatric damage in our cohort is comparable to data from developed countries. ${ }^{7,20,25,28}$

The main limitation in our study was our small sample size and the retrospective nature of our study. There may also have been underrecognition of NPSLE in our cohort of JSLE patients, given that many of the features are non-specific. In addition, cognitive and subtle mood disorders may be missed in the absence of routine objective testing of all JSLE patients as similarly highlighted in other studies. The strength of our cohort is that we are the sole tertiary referral center for the whole country and are therefore able to give a fairly accurate reflection of this condition in Malaysia.

Neuropsychiatric lupus is rare in children but can be present in any patient with JSLE. It has a higher incidence within the first year of JSLE diagnosis and is associated with high disease activity. Diagnosis of NPSLE remains a challenge as a normal CSF finding does not exclude its presence, and MRI abnormalities 
when present are often nonspecific. Although severe neurological manifestations are common, majority have good prognosis with complete neurological recovery following early recognition and intensive treatment.

\section{Acknowledgements}

The authors wish to thank the Director General of Health, Ministry of Health Malaysia for allowing their facilities to be used for this research.

\section{Author contribution}

The authors confirm contribution to the paper as follows: study conception and design: SCL, SPT; data collection: SCL, YL, BJ, RFAK; analysis and interpretation of results: SCL, SPT, YL, BJ, RFAK; draft manuscript preparation: SCL, YL, SPT. All authors reviewed the results and approved the final version of the manuscript.

\section{Source of funding}

This is an investigator-initiated research and received no external funding.

\section{Conflict of interest}

The authors declare that they have no conflict of interest.

\section{REFERENCES}

1. Smith PP, Gordon C. Systemic lupus erythematosus: clinical presentations. Autoimmun Rev 2010; 10: 4345 .

2. Duarte-Delgado NP, Vásquez G, OrtizReyes BL. Blood-brain barrier disruption and neuroinflammation as pathophysiological mechanisms of the diffuse manifestations of neuropsychiatric systemic lupus erythematosus. Autoimmun Rev 2019; 18: 426-432.

3. Haji Muhammad Ismail Hussain I, Loh WF, Sofiah A. Childhood cerebral lupus in an Oriental population. Brain Dev 1999; 21: 229-235.
4. Yu HH, Lee JH, Wang LC, Yang YH, Chiang BL. Neuropsychiatric manifestations in pediatric systemic lupus erythematosus: a 20-year study. Lupus 2006; 15: 651-657.

5. Khajezadeh MA, Zamani G, Moazzami B, Nagahi Z, Mousavi-Torshizi M, Ziaee V. Neuropsychiatric involvement in juvenile-onset systemic lupus erythematosus. Neurol Res Int 2018; 2018: 2548142.

6. Kivity S, Agmon-Levin N, Zandman-Goddard G, Chapman J, Shoenfeld Y. Neuropsychiatric lupus: a mosaic of clinical presentations. BMC Med 2015; 13: 43 .

7. Benseler SM, Silverman ED. Neuropsychiatric involvement in pediatric systemic lupus erythematosus. Lupus 2007; 16: 564-571.

8. Olfat MO, Al-Mayouf SM, Muzaffer MA. Pattern of neuropsychiatric manifestations and outcome in juvenile systemic lupus erythematosus. Clin Rheumatol 2004; 23: 395-399.

9. Sit JKK, Chan WKY. Risk factors for damage in childhood-onset systemic lupus erythematosus in Asians: a case control study. Pediatr Rheumatol Online J 2018; 16: 56.

10. Suwanpakdee P, Nabangchang C, Lumpaopong A. Neuropsychiatric manifestations in pediatric systemic lupus erythematosus. J Med Assoc Thai 2017; 100: 1196.

11. Petri M, Orbai AM, Alarcón GS, et al. Derivation and validation of the Systemic Lupus International Collaborating Clinics classification criteria for systemic lupus erythematosus. Arthritis Rheum 2012; 64: 2677-2686.

12. The American College of Rheumatology nomenclature and case definitions for neuropsychiatric lupus syndromes. Arthritis Rheum 1999; 42: 599-608.

13. Gladman DD, Ibañez D, Urowitz MB. Systemic lupus erythematosus disease activity index 2000. J Rheumatol 2002; 29: 288-291.

14. AlE'ed A, Vega-Fernandez P, Muscal E, et al; CARRA Neuropsychiatric Systemic Lupus Erythematosus Working Group. Challenges of diagnosing cognitive dysfunction with neuropsychiatric systemic lupus erythematosus in childhood. Arthritis CareRes (Hoboken) 2017; 69: 1449-1459.

15. Harel L, Sandborg C, Lee T, von Scheven E. Neuropsychiatric manifestations in pediatric systemic lupus erythematosus and association with antiphospholipid antibodies. J Rheumatol 2006; 33: 1873-1877. 
16. de Amorim JC, Frittoli RB, Pereira D, et al. Epidemiology, characterization, and diagnosis of neuropsychiatric events in systemic lupus erythematosus. Expert Rev Clin Immunol 2019; 15: 407-416.

17. Demirkaya E, Bilginer Y, Aktay-Ayaz N, et al. Neuropsychiatric involvement in juvenile systemic lupus erythematosus. Turk J Pediatr 2008; 50: 126131.

18. Faria R, Gonçalves J, Dias R. Neuropsychiatric systemic lupus erythematosus involvement: towards a tailored approach to our patients? Rambam Maimonides Med J 2017; 8: e0001.

19. Bertsias GK, Boumpas DT. Pathogenesis, diagnosis and management of neuropsychiatric SLE manifestations. Nat Rev Rheumatol 2010; 6: 358-367.

20. Magro-Checa C, Zirkzee EJ, Huizinga TW, SteupBeekman GM. Management of neuropsychiatric systemic lupus erythematosus: current approaches and future perspectives. Drugs 2016; 76: 459-483.

21. Ho RC, Thiaghu C, Ong $\mathrm{H}$, et al. A meta-analysis of serum and cerebrospinal fluid autoantibodies in neuropsychiatric systemic lupus erythematosus. Autoimmun Rev 2016; 15: 124-138.

22. Al-Obaidi M, Saunders D, Brown S, et al. Evaluation of magnetic resonance imaging abnormalities in juvenile onset neuropsychiatric systemic lupus erythematosus. Clin Rheumatol 2016; 35: 2449-2456.
23. Ainiala H, Dastidar P, Loukkola J, et al. Cerebral MRI abnormalities and their association with neuropsychiatric manifestations in SLE: a population-based study. Scand J Rheumatol 2005; 34: 376-382.

24. Govoni M, Hanly JG. The management of neuropsychiatric lupus in the 21st century: still so many unmet needs? Rheumatology (Oxford) 2020; 59(Suppl5): v52-v62.

25. Lopes SRM, Gormezano NWS, Gomes RC, et al; Brazilian Childhood-onset Systemic Lupus Erythematosus Group. Outcomes of 847 childhoodonset systemic lupus erythematosus patients in three age groups. Lupus 2017; 26: 996-1001.

26. Mansour HE, Habeeb RA, El-Azizi NO, et al. Electroencephalography in systemic lupus erythematosus patients with neuropsychiatric manifestations. Egypt J Int Med 2020; 32: 11.

27. Bertsias GK, Ioannidis JPA, Aringer M, et al. EULAR recommendations for the management of systemic lupus erythematosus with neuropsychiatric manifestations: report of a task force of the EULAR standing committee for clinical affairs. Ann Rheum Dis 2010; 69: 2074-2082.

28. Groot N, Shaikhani D, Teng YKO, et al. Longterm clinical outcomes in a cohort of adults with childhood-onset systemic lupus erythematosus. Arthritis Rheumatol 2019; 71: 290-301. 